\title{
Diabetic Ketoacidosis, Hypertriglyceridemia and Abdominal Pain due to Acute Pancreatitis Complicated by Non-immune Haemolytic Anaemia
}

\author{
Monica L Joustra ${ }^{1}$, Janneke J Raidt ${ }^{1}$, Florens Droog ${ }^{2}$, Thiemo F Veneman ${ }^{2}$ \\ ${ }^{1}$ Department of Internal Medicine, Ziekenhuisgroep Twente (Hospital Group Twente), Almelo, The Netherlands \\ ${ }^{2}$ Department of Intensive Care Medicine, Ziekenhuisgroep Twente (Hospital Group Twente), Almelo, The Netherlands
}

Doi: 10.12890/2020_002085 - European Journal of Case Reports in Internal Medicine - ๑ EFIM 2020

Received: 20/10/2020

Accepted: 04/11/2020

Published: 09/12/2020

How to cite this article: Joustra ML, Raidt JJ, Droog F, Veneman TF. Diabetic ketoacidosis, hypertriglyceridemia and abdominal pain due to acute pancreatitis complicated by non-immune haemolytic anaemia. EJCRIM 2020;7: doi:10.12890/2020_002085.

Conflicts of Interests: The Authors declare that there are no competing interests.

This article is licensed under a Commons Attribution Non-Commercial 4.0 License

\section{ABSTRACT}

The triad of diabetic ketoacidosis, acute pancreatitis and hypertriglyceridemia is a rare phenomenon, with mortality rates of up to $80 \%$. A unique characteristic of the described case is the co-occurrence of non-immune haemolytic anaemia (NIHA) with the complex triad. It is suggested that this presentation is secondary to hyperlipidemia which leads to increased fragility of erythrocytes due to destabilization of red cell membranes. Supportive treatment with intravenous insulin and blood transfusions is the cornerstone of treatment.

\section{LEARNING POINTS}

- The enigmatic triangle of diabetic ketoacidosis (DKA), hypertriglyceridemia and acute pancreatitis is a rare phenomenon occurring in only $4 \%$ of DKA cases.

- This triad can be complicated by non-immune haemolytic anaemia secondary to hyperlipidemia, which leads to increased fragility of the erythrocyte due to destabilization of red cell membranes.

- Supportive treatment with intravenous insulin administration and blood transfusions is the cornerstone of treatment.

\section{KEYWORDS}

Acute pancreatitis, diabetes ketoacidosis, hypertriglyceridemia, haemolytic anaemia

\section{INTRODUCTION}

Diabetic ketoacidosis (DKA) is a severe and potentially lethal complication of diabetes mellitus (DM). It is commonly, but not exclusively, encountered in patients with type 1 DM. DKA is a state of absolute insulin deficiency which can result in hypertriglyceridemia. A triad of DKA, hypertriglyceridemia and acute pancreatitis has previously been described, and is a rare phenomenon ${ }^{[1]}$. The triad is referred to as 'the enigmatic triangle' and occurs in only $4 \%$ of DKA cases. A mortality rate of $80 \%$ has been described. The exact underlying pathophysiological mechanism is not fully understood: is DKA the cause of, or is it a complication of, acute pancreatitis?

In addition to this lethal triad, haemolysis also played an important role in the present case. The association of acute pancreatitis and haemolysis is rare. Haemolysis can trigger acute pancreatitis but can also be a consequence. Approximately $25 \%$ of cases of massive haemolysis are complicated by acute pancreatitis ${ }^{[2]}$.

In this report we will discuss the complex relationship between the lethal triad of DKA, acute pancreatitis and hypertriglyceridemia accompanied by haemolysis. 


\section{CASE DESCRIPTION}

A 31-year-old woman with a history of gestational diabetes and appendectomy presented with acute onset of abdominal pain, without nausea, vomiting or diarrhoea. On physical examination, she was afebrile and normotensive, with an increased respiratory rate of 36/min, hypoxemia and sinus tachycardia of 130/min. The abdomen was firm, with decreased bowel sounds and tenderness throughout the entire abdomen.

On admission, haemoglobin level, renal function and serum liver enzymes were normal (Table 1). Serum lipase was >600 U/I, and hyponatremia and hyperglycaemia were observed. Arterial blood gas analysis showed respiratory compensation for metabolic acidosis. Urinalysis was positive for ketones.

Abdominal ultrasound confirmed the diagnosis of acute pancreatitis. Because of persistent hypoxemia, the patient was admitted to the intensive care unit for respiratory support and further treatment of the DKA and pancreatitis. Additional laboratory results showed high plasma triglycerides as a potential cause of pancreatitis (Table 1). Antibodies against the 65 kDa isoform of GAD (GAD65) were not detectable, suggesting type 2 diabetes.

After 3 days of hospitalization, the patient developed normocytic anemia accompanied by decreased haptoglobin, suggesting haemolysis (Table 1). Reticulocytes were in the normal range (55×10\%/I; normal range $25-120)$. To differentiate between autoimmune haemolytic anemia (AIHA) and non-immune haemolytic anaemia (NIHA), a direct antiglobulin test (DAT, direct Coombs) was performed. The DAT was negative, indicating acquired NIHA. Erythrocyte microscopy showed normal erythrocyte morphology. Since schistocytes were absent, there was no evidence for microangiopathic haemolysis. Fibrinogen was elevated at $8.6 \mathrm{~g} / \mathrm{l}$ (normal range 2-4), von Willebrand factor activity was $>150 \%$ (normal range 50-150\%), factor VIII was 490\% (normal range 50-150\%), and ADAMSTS13 was normal. Furthermore, vitamin B12 (109 pmol/l) and folic acid (>445 nmol/l) were normal. Since no underlying cause for haemolysis was found, supportive treatment was initiated with multiple blood transfusions.

After 1 week, the haemoglobin level had stabilized. Six days after hospitalization, triglycerides had decreased to $2.3 \mathrm{mmol} / \mathrm{l}$. After $1 \mathrm{week}$ in the intensive care unit, the patient was transferred to the regular ward. Long-acting insulin was started. She recovered quickly and left the hospital in good clinical condition.

\section{DISCUSSION}

This case report describes a patient who initially presented with the triad DKA, acute pancreatitis and hypertriglyceridemia complicated by NIHA. The diagnosis of acute pancreatitis in patients with DKA can be challenging because the main initial complaint (abdominal pain) is a common feature in DKA and therefore not specific for pancreatitis. Furthermore, $25 \%$ of patients with DKA have elevated levels of serum amylase and lipase without the presence of clinical and/or radiological evidence for the diagnosis acute pancreatitis ${ }^{[3]}$. On the other hand, pancreatitis induced by hypertriglyceridemia in DKA can be accompanied by normal levels of amylase/lipase due to the lipemic content of the blood.

One of the main questions is whether DKA is the cause of, or a complication of, acute pancreatitis. In this specific case, we suspected that the DKA was the underlying cause of the pancreatitis. It is suggested that the insulin-deficient state in DKA promotes lipolysis which in turn results in the release of free fatty acids from adipose tissue and muscle, resulting in hypertriglyceridemia ${ }^{[4]}$. A high triglyceride level $(>11.3$ $\mathrm{mmol} / \mathrm{l}$ ) is an identifiable risk factor for the occurrence of triglyceride-induced pancreatitis. In this case, the initial plasma triglyceride level was $45.6 \mathrm{mmol} / \mathrm{l}$. In the literature, triglyceride levels are found to be normal after 3 days of treatment or fasting in triglyceride-induced pancreatitis.

A unique characteristic of this case was the co-occurrence of NIHA with the complex triad after 3 days of hospitalization. A few cases describing haemolysis as a complication of acute pancreatitis can be found in the literature ${ }^{[4]}$. On the other hand, massive haemolysis can trigger pancreatitis. It has been proposed that a proinflammatory state is stimulated due to the presence of large amounts of heme in the blood. It has also been reported that haemolysis can be a rare complication of hyperlipidemia, mainly hypertriglyceridemia ${ }^{[5]}$. It has been suggested that increased lipids may alter the composition of the erythrocyte membrane which in turn leads to increased fragility of the erythrocyte due to destabilization of red cell membranes (Fig. 1). Haemolyzed blood samples in a patient presenting with abdominal pain should prompt the clinician to consider the presence of hypertriglyceridemia-induced pancreatitis ${ }^{[6]}$.

In the present case, the DKA and the secondary triglyceride-induced pancreatitis were treated with intravenous insulin administration in combination with crystalline fluids. Six days after patient hospitalization, triglyceride levels had decreased due to the treatment for DKA, which subsequently halted the lipolysis and release of free fatty acids resulting in lowering of plasma triglycerides. Furthermore, lowering of the plasma triglycerides concentration improved the clinical presentation of the pancreatitis. Secondly, supportive treatment with blood transfusions was initiated to counter anemia due to haemolysis. After 1 week, the haemoglobin level had stabilized. 


\begin{tabular}{|c|c|c|c|c|c|}
\hline & On admission & +1 day & +2 days & +3 days & \\
\hline \multicolumn{6}{|l|}{ Haematology } \\
\hline Haemoglobin & 11.1 & 9.9 & 7.8 & 4.9 & $\mathrm{mmol} / \mathrm{l}$ \\
\hline Haematocrit & 0.5 & & & & $\mid / /$ \\
\hline MCV & & & & 84 & $f l$ \\
\hline Haptoglobin & & & & $<0.2$ & $g / l$ \\
\hline C-reactive protein & 116 & 360 & 500 & 395 & $m g / l$ \\
\hline Leukocytes & 7.34 & 8.06 & 8.19 & 10.1 & $10^{9} / 1$ \\
\hline Thrombocytes & 216 & 191 & 175 & 154 & $10^{9} / 1$ \\
\hline \multicolumn{6}{|l|}{ Chemistry } \\
\hline Bilirubin & $<17$ & $<17$ & $<17$ & 24 & $\mu \mathrm{mol} / \mathrm{l}$ \\
\hline Alkaline phosphatase & 52 & $31^{*}$ & 35 & 45 & $U / I$ \\
\hline Gamma GT & 165 & 31 & 37 & 66 & U/I \\
\hline ASAT & $<5$ & $105^{*}$ & $96^{*}$ & 139 & $U / I$ \\
\hline ALAT & $<5$ & $12^{*}$ & 12 & 12 & $U / I$ \\
\hline$L D$ & & $1246^{*}$ & $1704^{*}$ & $2407^{*}$ & $U / I$ \\
\hline Urea & 2.8 & 5.6 & 7.3 & 8.9 & $\mathrm{mmol} / \mathrm{l}$ \\
\hline Creatinine & 58 & 110 & 110 & 158 & $\mu \mathrm{mol} / \mathrm{l}$ \\
\hline eFGR CKD-epi (per $\left.1.73 \mathrm{~m}^{2}\right)$ & $>90$ & 58 & 57 & 37 & $\mathrm{ml} / \mathrm{min} / 1.73 \mathrm{~m}^{2}$ \\
\hline Sodium & 120 & 130 & 126 & 130 & $\mathrm{mmol} / \mathrm{l}$ \\
\hline Potassium & 4.6 & 4.7 & 4.6 & 3.4 & $\mathrm{mmol} / \mathrm{l}$ \\
\hline Lipase & $>600$ & & & & $U / I$ \\
\hline Glucose & 21.2 & 17 & 11.4 & 9.5 & $\mathrm{mmol} / \mathrm{l}$ \\
\hline HCG & $<1$ & & & & $U / /$ \\
\hline Triglycerides & & 45.6 & & & $\mathrm{mmol} / \mathrm{l}$ \\
\hline pH & 7.23 & 7.32 & 7.31 & 7.32 & \\
\hline $\mathrm{pCO}_{2}$ & 3 & 3.3 & 4.5 & 4.9 & $\mathrm{kPa}$ \\
\hline Bicarbonate & 9 & 13 & 17 & 19 & $\mathrm{mmol} / \mathrm{l}$ \\
\hline Base excess & -16 & $\neg 11.2$ & $\neg 8.6$ & $\neg 6.5$ & $\mathrm{mmol} / \mathrm{l}$ \\
\hline $\mathrm{pO}_{2}$ & 12.3 & 16.4 & 17.3 & 9.5 & $\mathrm{kPa}$ \\
\hline \multicolumn{6}{|l|}{ Urinalysis } \\
\hline Erythrocytes & $10-50$ & & & & $/ \mu l$ \\
\hline Leukocytes & $<10$ & & & & $/ \mu l$ \\
\hline Glucose & $>20$ & & & & $\mathrm{mmol} / \mathrm{l}$ \\
\hline Ketones & $>5$ & & & & $\mathrm{mmol} / \mathrm{l}$ \\
\hline Total protein & $0.5-1.5$ & & & & $g / l$ \\
\hline Nitrite & Negative & & & & \\
\hline$p H$ & 5.0 & & & & \\
\hline
\end{tabular}

Table 1. Laboratory results. Red colour indicates a high value, blue colour indicates a low value. ${ }^{*}$ Too high due to haemolysis 


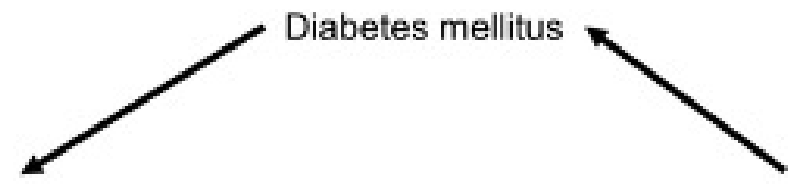

Diabetic ketoacidosis

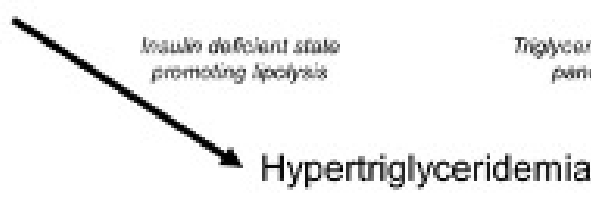

Acute pancreatitis

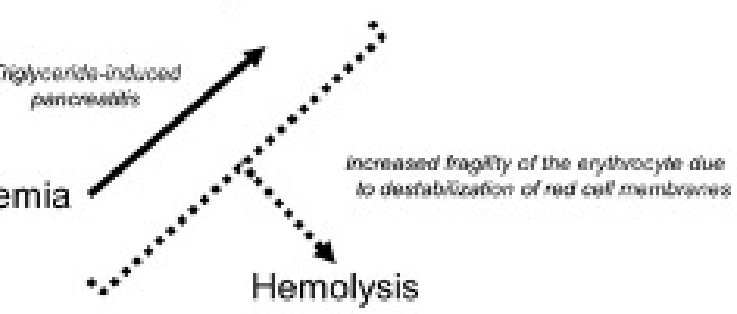

Figure 1. Complex interrelationship of the various disorders

To our knowledge, this is the first report of a patient presenting with DKA and severe hypertriglyceridemia resulting in acute pancreatitis which was complicated by NIHA. As discussed, in each case the causes and secondary consequences should be evaluated as several different scenarios are possible. The clinician should consider possible devastating consequences, such as acute pancreatitis due to hypertriglyceridemia or haemolysis, which benefit from intravenous insulin therapy and supportive treatment.

\section{REFERENCES}

1. Roberto Simons-Linares C, Jang S, Sanaka M, Bhatt A, Lopez R, Vargo J, et al. The triad of diabetes ketoacidosis, hypertriglyceridemia and acute pancreatitis. How does it affect mortality and morbidity?: A 10-year analysis of the National Inpatient Sample. Medicine 2019;98(7):e14378.

2. Druml W, Laggner AN, Lenz K, Grimm G, Schneeweiss B. Pancreatitis in acute hemolysis. Ann Hematol 1991;63(1):39-41.

3. Yadav D, Nair S, Norkus EP, Pitchumoni CS. Nonspecific hyperamylasemia and hyperlipasemia in diabetic ketoacidosis: incidence and correlation with biochemical abnormalities. Am J Gastroenterol 2000;95(11):3123-3128.

4. Kota SK, Krishna SV, Lakhtakia S, Modi KD. Metabolic pancreatitis: etiopathogenesis and management. Indian J Endocrinol Metab 2013;17:799-805.

5. Dimeski G, Mollee P, Carter A. Increased lipid concentration is associated with increased hemolysis. Clin Chem 2005;51(12):2425.

6. Tariq R, Khanna S. Hemolyzed blood as a clue to the diagnosis of abdominal pain. Intern Emerg Med 2016;11(4):609-610. 